\title{
BMJ Open Are neuromuscular adaptations present in people with recurrent spinal pain during a period of remission? A protocol for a systematic review
}

\author{
Valter Devecchi, Alessio Gallina, Nicola R Heneghan (D), Alison B Rushton (D), \\ Deborah Falla (1)
}

To cite: Devecchi V, Gallina A, Heneghan NR, et al. Are neuromuscular adaptations present in people with recurrent spinal pain during a period of remission? A protocol for a systematic review. BMJ Open 2019:9:e033276. doi:10.1136/ bmjopen-2019-033276

- Prepublication history and additional material for this paper are available online. To view these files, please visit the journal online (http://dx.doi org/10.1136/bmjopen-2019033276).

Received 29 July 2019 Revised 02 October 2019 Accepted 25 November 2019

A) Check for updates

(C) Author(s) (or their employer(s)) 2019. Re-use permitted under CC BY-NC. No commercial re-use. See rights and permissions. Published by BMJ.

Centre of Precision

Rehabilitation for Spinal Pain (CPR Spine), School of Sport, Exercise and Rehabilitation Sciences, College of Life and Environmental Sciences, University of Birmingham, Birmingham, UK

Correspondence to Professor Deborah Falla; d.falla@bham.ac.uk

\section{ABSTRACT}

Introduction The course of spinal pain (neck or low back pain) is often described as episodic and intermittent, with more than one-third of people continuing to experience episodic symptoms 1 year after first onset. Although ongoing neuromuscular adaptations could contribute to recurrent episodes of pain, no systematic review has synthesised evidence of ongoing neuromuscular changes in people with recurrent spinal pain during a period of symptom remission.

Methods and analysis This protocol is developed and reported following the Preferred Reporting Items for Systematic Reviews and Meta-Analyses-P, the Update of the Cochrane Back and Neck Group guidelines and the Methodological Expectations of Cochrane Intervention Reviews. PubMed, Web of Science, MEDLINE, EMBASE, CINAHL, ZETOC, Google Scholar, grey literature sources and key journals will be searched up to September 2019. Observational studies investigating neuromuscular changes in people with recurrent spinal pain during a period of remission will be included. Neuromuscular function will be considered under five outcome domains of muscle activity, spine kinematics, muscle properties, sensorimotor control and neuromuscular performance. Two independent reviewers will search, screen studies, extract data and assess risk of bias (Newcastle-Ottawa Scale). Data will be synthesised per outcome domain. Where clinical and methodological homogeneity across studies exists, a random-effects meta-analysis will be conducted. Otherwise, results will be synthesised narratively. The overall quality of evidence will be assessed using the Grading of Recommendations, Assessment, Development and Evaluation guidelines. Ethics and dissemination Findings of this review may aid the identification of factors that could contribute to spinal pain recurrence and aid the development of interventions for secondary prevention aimed at the restoration of optimal neuromuscular function. The results will be submitted for publication in a peer-reviewed journal and presented at conferences. No ethical approval was required.

PROSPERO registration number CRD42019141527.

\section{INTRODUCTION}

Low back and neck pain (spinal pain) continue to be the leading cause of disability
Strengths and limitations of this study

- This will be the first systematic review to synthesise evidence of neuromuscular adaptations in people with recurrent spinal pain (neck or low back pain) during a period of remission.

- This protocol is written following the recommendations reported in version 6 of the Cochrane Handbook for Systematic Reviews of Interventions that will be published in mid-2019 (second edition of the Handbook).

- Neuromuscular changes will be evaluated considering a broad range of outcome domains, specifically muscle activity, spine kinematics, muscle properties, sensorimotor control and neuromuscular performance.

- In accordance with the Grading of Recommendations Assessment, Development, and Evaluation approach, the inclusion of observational studies reduces the quality of evidence.

worldwide. ${ }^{1}$ The number of years lived with disability has increased by more than $50 \%$ since 1990 , creating a 'spinal pain epidemic'. ${ }^{12}$ In particular, people aged between 25 and 64 years are most affected by spinal pain ${ }^{1}$ and thus the economic impact is substantial due to work absences and healthcare cost. ${ }^{3}$

Spinal pain is a particularly challenging condition since the prognosis is often poor following an acute episode and many people experience recurrent pain. ${ }^{4-6}$ For instance, more than $50 \%$ and $30 \%$ of patients experience further neck or low back pain episodes, respectively, within the first year following a first episode. ${ }^{78}$ Indeed, the course of spinal pain is often described as repeated episodes of pain, commonly across a lifetime, with varying degrees of recovery between episodes. Summarising 10 cohort studies, Kongsted $e t a t^{\beta}$ identified different patterns of low back pain progression, distinguishing between persistent, fluctuating, recurrent 
and single episode trajectories. Conceptually, it is also necessary to distinguish between new painful episodes characterising recurrent patterns ${ }^{10}$ and the notion of flare, a sudden and severe increase of symptoms affecting persistent or fluctuating conditions. ${ }^{11}$ Recurrent episodes of pain were described by de Vet $e t a l^{10}$ as periods of pain lasting for 1 day or more and separated by at least 1 month of symptom remission. Fluctuating patterns differ from recurrent pain because the former does not include period of remission, so patients continue to experience symptoms but with different level of intensity. ${ }^{9} 10$

Several factors may contribute to recurrent episodes of pain including ongoing maladaptive motor behaviour, neuromuscular deficits, impaired sensorimotor control and fear avoidance. ${ }^{12-15}$ More specifically, in people with spinal pain a number of common neuromuscular changes have been observed which have the potential to contribute to repeated episodes of pain. These include reduced muscle strength, endurance, ${ }^{16-19}$ range of motion, ${ }^{20}{ }^{21}$ increased muscle co-activation, ${ }^{22}{ }^{23}$ poorer control of the deeper muscles, ${ }^{24}{ }^{25}$ poorer proprioception and changes in the quality and variability of movement. ${ }^{26-29}$ Although these changes have been contextualised to be protective in nature ${ }^{30} 31$ and occur early following the onset of pain, ${ }^{32-34}$ they appear to be sustained beyond the acute phase $^{13536}$ and have the potential to contribute to chronicity and repeated painful episodes. ${ }^{12} 313738$ Although numerous studies have confirmed the presence of altered neuromuscular function in patients with chronic neck $^{1839}$ and back pain ${ }^{254041}$ and systematic reviews have been conducted to critically appraise these studies, and synthesise findings, ${ }^{21}{ }^{42-44}$ no systematic review has been conducted to evaluate evidence of neuromuscular changes during a period of remission in people with recurrent spinal pain. The assessment of patients during a period of remission allows to investigate neuromuscular function without pain interference.

The aim of this systematic review is to summarise the current literature to determine the evidence supporting the existence of ongoing neuromuscular adaptations in people with recurrent spinal pain during a period of remission. It is expected that this synthesis of evidence will influence clinical practice by highlighting the need to manage and restore neuromuscular function in addition to symptom suppression and will promote the relevance of interventions for secondary prevention.

\section{METHODS}

The protocol of this review has been developed following the update of the Cochrane Back and Neck Group guidelines ${ }^{45}$ the Methodological Expectations of Cochrane Intervention Reviews (MECIR) ${ }^{46}$ and the Preferred Reporting Items for Systematic Reviews and Meta-Analyses (PRISMA) Protocols (online supplementary file 1). ${ }^{47}$

\section{Eligibility criteria}

In order to detail the main elements of the systematic review, the PICOS framework will be used where $\mathrm{P}-\mathrm{Popu}-$ lation, I - Indicator, C - Comparator, O - Outcome(s) and $\mathrm{S}$ - Study design. ${ }^{47} 48$

\section{Population}

Adults (age $\geq 18$ years old) with recurrent non-specific spinal pain (neck or low back pain) are the population of interest and adults without a history of spinal pain represent the control group. For the purpose of this review and in order to avoid the exclusion of relevant articles, the definition provided by de Vet $e t a l^{10}$ will be partially considered due to the heterogeneity identified during the scoping search. Therefore, for the recurrent spinal pain group, we will consider studies where the individuals have experienced episodes of spinal pain on at least two occasions during the previous year. ${ }^{10}$ Participants with injury that resulted in a fracture or radiating, neuropathic pain will be excluded. Pain intensity reported before the outcome assessment will not represent an element of exclusion but it will be considered for further subgroup analysis (no pain vs minimal pain) as the initial scoping search identified that some studies define a period of remission to be minimal pain rather than absence of pain.

\section{Indicator}

The indicator of interest is the use of:

- Surface electromyography (EMG), intramuscular EMG, ultrasound and functional magnetic resonance imaging (fMRI) to measure axial muscle activity/ behaviour. ${ }^{4-52}$

- Motion analysis systems (eg, optoelectronic systems, inertial measurement unit sensors) to quantify spine kinematics. $^{53}$

- Ultrasound and MRI to investigate muscle properties. $^{5455}$

- Dynamometry and performance testing to assess strength and endurance of axial muscles.

Therefore, all muscles (superficial and deep) that act on the spine and movements of the lumbar, thoracic and cervical regions will be considered.

\section{Comparison}

Between-group comparisons will be analysed considering people with recurrent spinal pain during a period of remission and healthy subjects without a history of spinal pain (neck or low back pain). Moreover, studies must include comparisons related to axial muscle activity, spine kinematics, sensorimotor control, muscle properties, strength and endurance during rest or tasks (static and/or dynamic).

\section{Outcomes}

The outcomes of interest are chosen based on the purpose of the review and the theoretical framework that has been suggested for people with spinal pain. ${ }^{31} 3738$

Moreover, in accordance with the literature neuromuscular adaptations selected can influence long-term 
Table 1 Outcome domains

\begin{tabular}{lll}
\hline Concept measured & Broad outcome domains & Narrow outcome domains \\
\hline Neuromuscular adaptations of the spine & Muscle activity & Amplitude of activity and its variability \\
& Spine kinematics & Timing of activation and its variability \\
& & Motor variability \\
& Sensorimotor control & Quality of movement \\
Muscle properties & Proprioception \\
& & Total cross-sectional area (CSA) \\
& Muscle CSA \\
& Neuromuscular performance & Strength fat infiltration \\
& & Endurance/fatigue
\end{tabular}

negative consequences and they could contribute to trigger future episodes of pain. ${ }^{31}$

The main concept investigated is the neuromuscular system; therefore, broad domains such as axial muscle activity, spinal kinematics, sensorimotor control, properties of the axial muscles and performance will be considered. In order to narrow the review focus, specific outcome domains are identified within the broad domains and are summarised in table 1 .

Outcome domains are operationalised and different outcome measures are considered as described in the following paragraphs.

Muscle activity domain will comprise muscle timing (during predicted and non-predicted perturbations) and muscle amplitude. The outcome measures that will be extracted to report amplitude and muscle timing with EMG are the average rectified value or root mean square and the onset of activity. Moreover, EMG variability related to the timing and amplitude of muscle activity will be considered. In the measurements obtained by ultrasound, the change of muscle thickness compared to rest value represents the outcome measure that will be used to report on changes in muscle activity. For fMRI measurements, parameters related to transverse relaxation time will be considered. ${ }^{51}$

Spine kinematics will comprise specific domains; motor variability, active range of motion and quality of movement (accuracy and smoothness) during functional tasks or tests. It is not possible to specify a priori the outcome measures because they depend on the tasks and instruments used.

Sensorimotor control of the spine will include proprioception investigated through specific tests (eg, repositioning and movement detection).

Muscle properties recorded with ultrasound or MRI include features such as the extent of fat infiltration, muscle thickness and total muscle cross-sectional area.

Strength and endurance values of the axial muscles investigated with dynamometry will be considered. Moreover, endurance related data obtained with the Borg Scale or EMG features (mean frequency or median frequency) will be included.

\section{Study design}

Based on the scoping search, observational studies will be considered because they address the PICOS framework specified in the review question.

\section{Information sources}

The search will be conducted from inception to 1 September 2019. Databases will include MEDLINE (OVID interface), EMBASE (OVID interface), CINAHL (EBSCO interface), ZETOC and Google Scholar. Moreover, specific Internet sites will be searched: PubMed, Web of Science and Cochrane Back and Neck Review Group.

The search process will be conducted using medical subject headings (MESH) where appropriate and relevant text words associated with the concepts of this review. The search strategy will be done operationalising the PICOS framework and the main concepts that will be considered are related to the population and outcomes of interest. Words associated with them will be linked with the Boolean terms AND/OR:

POPULATION:

'Recurrent' (using OR for all possible synonyms, such as intermittent, history of, episodes)

AND

'Spinal pain' (using OR for all spine regions)

AND INDICATOR:

'electromyography' OR 'motion analysis' OR 'dynamometer' (using OR for all possible synonyms and instruments used)

\section{AND OUTCOMES:}

'Spine kinematics' OR 'muscle activity' OR 'sensorimotor control' OR 'muscle properties' OR 'performance' (words/synonyms related to these concepts will be used)

Hand-searching will be conducted based on the results of the scoping search and including journals relevant for this review topic, specifically the Journal of Electromyography and Kinesiology, Clinical Biomechanics, The Clinical Journal of Pain, Spine, Musculoskeletal Science and Practice, and the Journal of Orthopaedic and Sports Physical Therapy. The eligibility of manuscripts included with hand searching will be defined using the PICOS framework. Relevant authors in the field will be contacted to obtain information about 
unpublished or ongoing studies. In order to reduce the risk of publication bias, grey literature will be included considering the British National Bibliography for report literature, OpenGrey and dissertation abstracts. In accordance with the MECIR standards ${ }^{46}$ reference lists of included studies and relevant systematic reviews will be checked for any further studies.

\section{Search strategy}

The search will not be restricted by date, region or language limits. The specific search strategy has been defined in accordance with a Health Sciences Librarian with review searching experience. An example of the search strategy (draft MEDLINE search - Ovid interface) is reported in online supplementary file 2 . Searching on MEDLINE (OVID interface) will be conducted using MESH. Based on other database selected, relevant terms will be adapted but search strategy consistency will be ensured.

\section{Data management}

EndNote V.X9 (Clarivate Analytics) will be used to manage the data, including citations, abstracts and full-text of relevant studies. A reviewer (VD) will upload studies during the searching process and duplicates will be removed. When the list of searched studies is completed, reviewers (VD and AG) will start the screening process. Full text of the papers that will be considered potentially eligible will be stored in EndNote V.X9.

\section{Selection process}

Based on the eligibility criteria, an electronic screening tool will be created and piloted. During the first stage of the screening process, two independent reviewers (VD and AG) will assess titles and abstracts against the eligibility criteria. In an event of disagreement, reviewers will first attempt to resolve through discussion. If no consensus can be reached, a third reviewer (DF) will mediate the process. After the first screening process, full-text records will be obtained for potentially eligible studies. The full text screening will be conducted by two reviewers (VD and AG). During both stages of the screening process the agreement between the two reviewers will be assessed using the kappa statistic. The PRISMA flow diagram will used to summarise the study selection process. ${ }^{47}$

\section{Data extraction process}

Data extraction will be performed independently by two reviewers $(\mathrm{VD}, \mathrm{AG})$ using a data extraction form developed and piloted a priori on a sub-group of studies. The data extraction form and table 2 will be customised based on the recommendations provided by Li $e t a l,{ }^{56}$ considering the eligibility criteria and aim of the review. If discrepancies between reviewers arise, they will be resolved by discussion. A third reviewer (DF) will be consulted to mediate where necessary. The authors of the primary studies will be contacted if the description of methods or data reporting is unclear. Duplicate publications will be identified in order to reduce bias that could influence

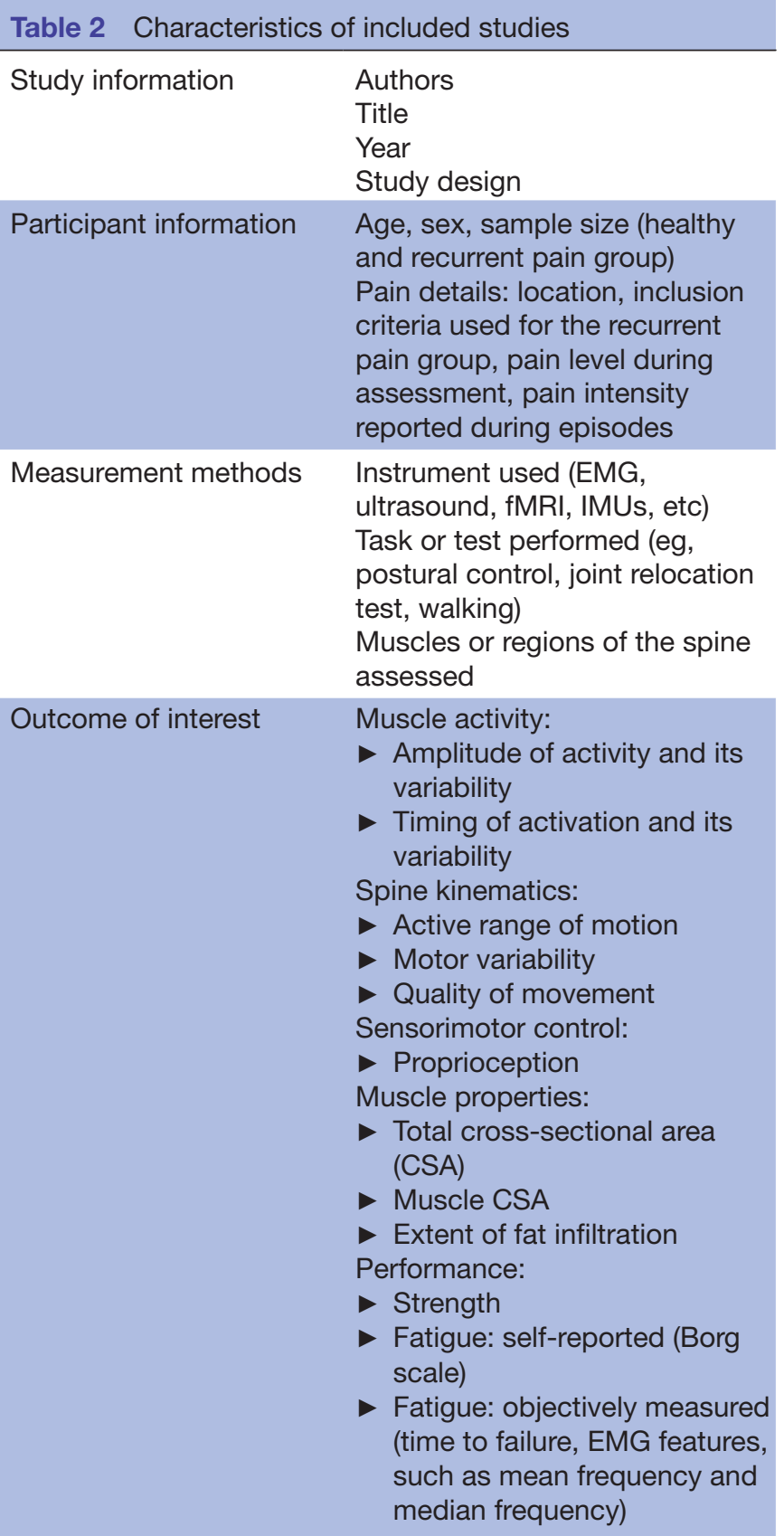

EMG, electromyography; fMRI, functional magnetic resonance imaging; IMU, inertial measurement unit.

quantitative results. Multiple reports of the same study will be collated, and a specific report will be chosen. The decision of the selected report will be justified.

In accordance with McKenzie et al, ${ }^{57}$ data extraction and synthesis will be simplified following specific criteria:

- When an outcome of interest is measured more than one time, the first assessment will be considered. Baseline measurements for cross-sectional comparisons with the healthy control group will be extracted in longitudinal studies or clinical trials.

- When an outcome of interest is measured with more than one tool, data will be selected based on the properties (reliability and validity) of the instrument. 
- Multiplicity of outcomes of the same domain (eg, strength of different muscles) will be reported and specific methods will be applied during data synthesis (see data synthesis section).

- Studies will be grouped based on the main outcome domain investigated; moreover, clinical and methodological diversity across studies will be analysed (see data synthesis section).

\section{Data items}

Study characteristics will include details of participant, outcomes, outcome measures, study design and they are reported in table 2 .

In order to reduce the impact of reporting bias, study authors will be contacted to obtain unpublished data or to clarify ambiguous results. However, if any uncertainty remains or the information provided by the author modifies the eligibility of the study, the paper will be excluded and a specific explanation will be provided. Data will be extracted from the control and recurrent pain group. If more than two groups are investigated in a study, data will be collected only from the healthy control group and the one that meets the eligibility criteria.

\section{Risk of bias}

Two independent reviewers (VD, AG) will appraise the included studies using the Newcastle-Ottawa Scale (NOS) ${ }^{58}$ Discrepancies will be resolved with discussion. The risk of bias tool has been chosen based on the review question and the design of the primary studies detected during the scoping search (observational). There is not a firm consensus about the more suitable risk of bias tool for observational studies and health assessment groups have provided poorly defined recommendations. ${ }^{5960}$ For example, in a recent review Quigley et $a b^{60}$ identified 48 critical appraisal tools for non-randomised studies. Given its strengths, the NOS has been the most used tool in non-randomised studies; especially since it is quick to complete, adaptable, validated and usable in all kind of observational studies. ${ }^{586162}$ Moreover, it assesses bias in the more relevant components of the studies included in this review; sample and outcomes. Considering the strengths of the NOS described previously, the case-control version will be used. ${ }^{58}$ The scale consists of three dimensions (selection, comparability, exposure) and eight items overall. The included studies will be assessed following the specific guidelines ${ }^{58}$ and a maximum of nine stars will be assigned to each paper ( $0-3=$ poor quality, $4-7=$ fair quality, 8-9=good quality). A table indicating the risk of bias in each study will be reported; moreover, detailed information for each dimension assessed by the NOS will be provided.

\section{Data synthesis}

Results will be synthesised following the framework developed by McKenzie $e t a l{ }^{63}$ Characteristics of each included study will be reported in a specific table. Then, studies and results will be grouped based on the main outcome domain investigated (muscle activity, spine kinematics, sensorimotor control, muscle properties, axial muscle performance) in the 'Major Findings' table. Moreover, data available for synthesis will be collected (mean and $\mathrm{SD})$. The difference between healthy and recurrent spinal pain group will be reported using the standardised mean difference and $95 \% \mathrm{CI}$; data will be obtained directly from the paper or calculated using the available information. Where relevant data are missing, the authors of the primary study will be contacted.

Random-effects meta-analysis (in accordance with the Cochrane Back and Neck Group) ${ }^{45}$ will be considered for a limited number of outcomes in order to reduce the risk of type I error (due to statistical multiplicity) ${ }^{64}$; therefore, the following outcomes are selected: muscle activity, muscle timing (during a postural perturbation), range of movements (including their variability) and muscle strength. These outcomes were selected considering the feasibility of their assessment in clinical practice and the theoretical framework of motor adaptation to pain. ${ }^{31}{ }^{38}$ Briefly, the features considered appear to be adopted with a protective purpose but may still be present during a period of remission (potentially contributing to long-term negative consequences). ${ }^{31} 38$

A separate meta-analysis will be conducted for each selected outcome or dependent variable where possible.

In order to decide whether results can be summarised with statistical synthesis, heterogeneity across studies will be explored. First, clinical and methodological diversity across studies will be analysed by the two reviewers considering the following elements:

- Muscle or region of the spine investigated (lumbar, thoracic or cervical).

- Task performed.

- Outcome measure reported (for muscle amplitude outcomes only normalised value will be considered).

Whether clinical and methodological homogeneity across studies investigating the same outcome domain will be sufficient, statistical heterogeneity will be analysed. Disagreement between reviewers will be resolved by discussion and if it will persist a third reviewer (DF) will be consulted.

Based on the scoping search, a large number of studies included a small sample size, thus in accordance to Higgins $e t a l,{ }^{65}$ the Cochran's $Q$ test will provide low power in the detection of significant heterogeneity. Therefore, the amount of inconsistency between studies will be assessed using the $\mathrm{I}^{2}$ statistic. ${ }^{66}$ If heterogeneity will be substantial $\left(\mathrm{I}^{2}>50 \%\right)$, the results of the considered outcome will be described using the vote-counting procedure (direction of difference or no difference) and a narrative synthesis will be provided. ${ }^{67}$ Additionally, if an adequate number of studies will be included, ${ }^{68}$ subgroup analysis will be performed in meta-analysis splitting participants with recurrent spinal pain (neck or low back pain) in two subgroups-subjects with and without minimal pain during the assessment. This method will be used in order to explore whether the presence of 
any pain influences motor control features. Subgroup comparison will be investigated using the fixed-effects model (between subgroups) described by Borenstein and Higgins. ${ }^{69}$

All the outcome results not included in the statistical synthesis will be described, when possible, with the votecounting procedure or narratively ${ }^{67}$; moreover, in the group of people with recurrent spinal pain, differences in findings between asymptomatic and symptomatic subjects during the assessment will be reported. The narrative synthesis will be conducted following specific guidance and the steps provided by the Cochrane Consumers and Communication Review Group. ${ }^{70} 71$

Results will be shown based on the method of synthesis (quantitative or qualitative) and grouped considering the outcome domains. Risk of bias will not represent elements of restriction in the presentation of findings. Risk of publication bias will be analysed adopting the search strategies described in the information source section; particularly, results from references different to peerreviewed articles will be included, as described by Page et $a l .{ }^{72}$ For example, hand searching of grey literature on trial registers will be conducted and notable authors will be contacted to obtain information on unpublished data or ongoing works.

\section{Confidence in cumulative estimate}

Quality of findings (certainty) will be assessed using the Grading of Recommendations Assessment, Development, and Evaluation (GRADE) approach. ${ }^{73}$ The GRADE process is 'outcome centric'; therefore, the quality of evidence will be analysed for each outcome domain included in the PICOS. ${ }^{75}$ In accordance with GRADE and considering that it is not possible to manipulate the independent variable (pain remission/no history of pain), the observational studies included will be considered a lowquality source of evidence. ${ }^{73}$ After this initial consideration, the certainty of evidence for each outcome across studies can be increased or decreased following specific criteria. Risk of bias, inconsistency, indirectness, imprecision and publication bias are reasons for lower quality of evidence. ${ }^{76}$ On the other hand, large effect size, doseresponse gradient and plausible confounding biases that underestimate the effect size are reasons to upgrade the certainty of evidence for the considered outcome across studies. ${ }^{76}$ Based on these criteria, GRADE rates the level of certainty for each outcome in four categories: 'High', 'Moderate', 'Low' and 'Very Low'. ${ }^{76}$ The GRADE guidelines provide detailed information on the assessment of the quality of evidence for observational studies. ${ }^{74}$ For example, study limitations to consider in observational studies during the evaluation of the strength of evidence are suggested. ${ }^{77}$

\section{Patient and public involvement}

The protocol was presented within patient and public involvement meetings in order to discuss the topic and obtain feedback for the review and future projects.
Patients will not be involved in the analysis and data collection of the systematic review.

\section{Implication of results}

The natural course of spinal pain (neck or low back pain) is not straightforward and different patterns of pain progression have been described. A previous episode of pain is one of the most relevant factors for future pain recurrences. Evidence has shown the role and relation between neuromuscular changes and pain in persistent musculoskeletal disorders and recovery of neuromuscular function has become an important component in rehabilitation. However, the focus for the management of acute pain is limited to pain management and resolution rather than full functional rehabilitation. The results of this systematic review may identify relevant neuromuscular adaptations that could play a role in the recurrent nature of spinal pain with the potential to therefore influence clinical practice.

\section{ETHICS AND DISSEMINATION}

Ethical approval is not required for this project as it will not involve utilisation or publication of personal data. The results of this systematic review will be disseminated through publication in a peer-reviewed journal and where appropriate, be presented in national and/or international conferences.

Twitter Nicola R Heneghan @HeneghanNicola, Alison B Rushton@abrushton and Deborah Falla @Deb_Falla

Contributors VD and DF are responsible for the conception of the research question, development of the protocol and initial drafting of the manuscript. DF is lead supervisor of VD and ABR and NRH are co-supervisors. All supervisors have provided guidance on methodological decisions and proposed analyses. VD and AG will be the first and second reviewer. DF will be the third reviewer. All authors will contribute to data interpretation, conclusions and dissemination. All authors have read and subsequently approved the final manuscript. DF is the guarantor of the review.

Funding The authors have not declared a specific grant for this research from any funding agency in the public, commercial or not-for-profit sectors.

Competing interests None declared.

Patient consent for publication Not required.

Provenance and peer review Not commissioned; externally peer reviewed.

Open access This is an open access article distributed in accordance with the Creative Commons Attribution Non Commercial (CC BY-NC 4.0) license, which permits others to distribute, remix, adapt, build upon this work non-commercially, and license their derivative works on different terms, provided the original work is properly cited, appropriate credit is given, any changes made indicated, and the use is non-commercial. See: http://creativecommons.org/licenses/by-nc/4.0/.

\section{ORCID iDs}

Nicola R Heneghan http://orcid.org/0000-0001-7599-3674

Alison B Rushton http://orcid.org/0000-0001-8114-7669

Deborah Falla http://orcid.org/0000-0003-1689-6190

\section{REFERENCES}

1 Vos T, Allen C, Arora M, et al. Global, regional, and national incidence, prevalence, and years lived with disability for 310 diseases and injuries, 1990-2015: a systematic analysis for the global burden of disease study 2015. Lancet 2016;388:1545-602. 
2 Buchbinder R, van Tulder M, Öberg B, et al. Low back pain: a call for action. Lancet 2018;391:2384-8.

3 Dagenais S, Caro J, Haldeman S. A systematic review of low back pain cost of illness studies in the United States and internationally. Spine J 2008;8:8-20.

4 Hush JM, Lin CC, Michaleff ZA, et al. Prognosis of acute idiopathic neck pain is poor: a systematic review and meta-analysis. Arch Phys Med Rehabil 2011;92:824-9.

5 Hoy DG, Protani M, De R, et al. The epidemiology of neck pain. Best Pract Res Clin Rheumatol 2010;24:783-92.

6 Hoy D, Brooks P, Blyth F, et al. The epidemiology of low back pain. Best Pract Res Clin Rheumatol 2010;24:769-81.

7 Stanton TR, Henschke N, Maher CG, et al. After an episode of acute low back pain, recurrence is unpredictable and not as common as previously thought. Spine 2008;33:2923-8.

8 Carroll LJ, Hogg-Johnson S, van der Velde G, et al. Course and prognostic factors for neck pain in the general population: results of the bone and joint decade 2000-2010 Task force on neck pain and its associated disorders. Spine 2008;33:S75-82.

9 Kongsted A, Kent P, Axen I, et al. What have we learned from ten years of trajectory research in low back pain? BMC Musculoskelet Disord 2016;17:220.

10 de Vet HCW, Heymans MW, Dunn KM, et al. Episodes of low back pain: a proposal for uniform definitions to be used in research. Spine 2002;27:2409-16.

11 Costa N, Ferreira ML, Cross M, et al. How is symptom flare defined in musculoskeletal conditions: a systematic review. Semin Arthritis Rheum 2018:48:302-17.

12 Meier ML, Vrana A, Schweinhardt P. Low back pain: the potential contribution of supraspinal motor control and proprioception. Neuroscientist 2019;25:583-96.

13 MacDonald D, Moseley LG, Hodges PW. Why do some patients keep hurting their back? evidence of ongoing back muscle dysfunction during remission from recurrent back pain. Pain 2009;142:183-8.

14 Chou R, Shekelle P. Will this patient develop persistent disabling low back pain? JAMA 2010;303:1295-302.

15 Dubois J-D, Abboud J, St-Pierre C, et al. Neuromuscular adaptations predict functional disability independently of clinical pain and psychological factors in patients with chronic non-specific low back pain. J Electromyogr Kinesiol 2014;24:550-7.

16 Sanderson A, Martinez-Valdes E, Heneghan NR, et al. Variation in the spatial distribution of erector spinae activity during a lumbar endurance task in people with low back pain. $J$ Anat 2019;234:532-42.

17 Moreno Catalá M, Schroll A, Laube G, et al. Muscle strength and neuromuscular control in low-back pain: elite athletes versus general population. Front Neurosci 2018;12:436.

18 Lindstroem R, Graven-Nielsen T, Falla D. Current pain and fear of pain contribute to reduced maximum voluntary contraction of neck muscles in patients with chronic neck pain. Arch Phys Med Rehabil 2012:93:2042-8.

19 Edmondston S, Björnsdóttir G, Pálsson T, et al. Endurance and fatigue characteristics of the neck flexor and extensor muscles during isometric tests in patients with postural neck pain. Man Ther 2011;16:332-8

20 Dall'Alba PT, Sterling MM, Treleaven JM, et al. Cervical range of motion discriminates between asymptomatic persons and those with whiplash. Spine 2001;26:2090-4.

21 Laird RA, Gilbert J, Kent P, et al. Comparing lumbo-pelvic kinematics in people with and without back pain: a systematic review and metaanalysis. BMC Musculoskelet Disord 2014:15:229.

22 van Dieën JH, Cholewicki J, Radebold A. Trunk muscle recruitment patterns in patients with low back pain enhance the stability of the lumbar spine. Spine 2003;28:834-41.

23 Lindstrøm R, Schomacher J, Farina D, et al. Association between neck muscle coactivation, pain, and strength in women with neck pain. Man Ther 2011;16:80-6.

24 Falla DL, Jull GA, Hodges PW. Patients with neck pain demonstrate reduced electromyographic activity of the deep cervical flexor muscles during performance of the craniocervical flexion test. Spine 2004;29:2108-14.

25 Massé-Alarie $\mathrm{H}$, Flamand VH, Moffet $\mathrm{H}$, et al. Corticomotor control of deep abdominal muscles in chronic low back pain and anticipatory postural adjustments. Exp Brain Res 2012;218:99-109.

26 Alsultan F, Cescon C, De Nunzio AM, et al. Variability of the helical axis during active cervical movements in people with chronic neck pain. Clin Biomech 2019;62:50-7.

27 Meisingset I, Woodhouse A, Stensdotter A-K, et al. Evidence for a general stiffening motor control pattern in neck pain: a cross sectional study. BMC Musculoskelet Disord 2015;16:56.
28 Gizzi L, Röhrle O, Petzke F, et al. People with low back pain show reduced movement complexity during their most active daily tasks. Eur J Pain 2019;23:410-8.

29 Pranata A, Perraton L, El-Ansary D, et al. Lumbar extensor muscle force control is associated with disability in people with chronic low back pain. Clin Biomech 2017;46:46-51.

30 Moseley GL, Butler DS. Fifteen years of explaining pain: the past, present, and future. J Pain 2015;16:807-13.

31 Hodges PW, Tucker K. Moving differently in pain: a new theory to explain the adaptation to pain. Pain 2011;152:S90-8.

32 Gizzi L, Muceli S, Petzke F, et al. Experimental muscle pain impairs the synergistic modular control of neck muscles. PLoS One 2015;10:e0137844.

33 Hodges PW, Coppieters MW, MacDonald D, et al. New insight into motor adaptation to pain revealed by a combination of modelling and empirical approaches. Eur J Pain 2013;17:1138-46.

34 Shojaei I, Salt EG, Hooker Q, et al. Comparison of lumbo-pelvic kinematics during trunk forward bending and backward return between patients with acute low back pain and asymptomatic controls. Clin Biomech 2017:41:66-71.

35 Madeleine P. On functional motor adaptations: from the quantification of motor strategies to the prevention of musculoskeletal disorders in the neck-shoulder region. Acta Physiologica 2010;199:1-46.

36 Hodges P, van den Hoorn W, Dawson A, et al. Changes in the mechanical properties of the trunk in low back pain may be associated with recurrence. J Biomech 2009;42:61-6.

37 Van Dieën JP, Reeves NP, Kawchuk G, et al. Motor control changes in low-back pain: divergence in presentations and mechanisms. $J$ Orthop Sports Phys Ther 2018;12:1-24.

38 van Dieën JH, Flor H, Hodges PW. Low-Back pain patients learn to adapt motor behavior with adverse secondary consequences. Exerc Sport Sci Rev 2017:45:223-9.

39 Sjölander P, Michaelson P, Jaric S, et al. Sensorimotor disturbances in chronic neck pain-Range of motion, peak velocity, smoothness of movement, and repositioning acuity. Man Ther 2008;13:122-31.

40 Christe G, Redhead L, Legrand T, et al. Multi-Segment analysis of spinal kinematics during sit-to-stand in patients with chronic low back pain. J Biomech 2016;49:2060-7.

41 Falla D, Gizzi L, Tschapek M, et al. Reduced task-induced variations in the distribution of activity across back muscle regions in individuals with low back pain. Pain 2014;155:944-53.

42 Knox MF, Chipchase LS, Schabrun SM, et al. Anticipatory and compensatory postural adjustments in people with low back pain: a systematic review and meta-analysis. Spine J 2018;18:1934-49.

43 Moghaddas D, de Zoete RMJ, Edwards S, et al. Differences in the kinematics of the cervical and thoracic spine during functional movement in individuals with or without chronic neck pain: a systematic review. Physiotherapy. In Press 2019;105:421-33.

44 de Zoete RMJ, Osmotherly PG, Rivett DA, et al. Sensorimotor contro in individuals with idiopathic neck pain and healthy individuals: a systematic review and meta-analysis. Arch Phys Med Rehabil 2017;98:1257-71.

45 Furlan AD, Malmivaara A, Chou R, et al. 2015 updated method guideline for systematic reviews in the Cochrane back and neck group. Spine 2015;40:1660-73.

46 Chandler J, Lasserson T, Higgins JPT. Standards for the planning, conduct and reporting of updates of Cochrane Intervention Reviews. In: Higgins JPT, Lasserson T, Chandler J, eds. Methodological expectations of Cochrane intervention reviews. London: Cochrane, 2016.

47 Shamseer L, Moher D, Clarke M, et al. Preferred reporting items for systematic review and meta-analysis protocols (PRISMA-P) 2015 elaboration and explanation. BMJ 2015;349:g7647.

48 Smith V, Devane D, Begley CM, et al. Methodology in conducting a systematic review of systematic reviews of healthcare interventions. BMC Med Res Methodol 2011;11:15.

49 Farina D, Merletti R, Enoka RM. The extraction of neural strategies from the surface EMG: an update. J Appl Physiol 2014;117:1215-30.

50 Vasseljen O, Fladmark AM, Westad C, et al. Onset in abdomina muscles recorded simultaneously by ultrasound imaging and intramuscular electromyography. J Electromyogr Kinesiol 2009;19:e23-31.

51 Cagnie B, Elliott J, O'Leary S, et al. Muscle functional MRI as an imaging tool to evaluate muscle activity. J Orthop Sports Phys Ther 2011;41:896-903.

52 Hodges PW, Pengel LHM, Herbert RD, et al. Measurement of muscle contraction with ultrasound imaging. Muscle Nerve 2003;27:682-92.

53 Cuesta-Vargas Al, Galán-Mercant A, Williams JM. The use of inertial sensors system for human motion analysis. Phys Ther Rev 2010;15:462-73. 
54 Cagnie B, Derese E, Vandamme L, et al. Validity and reliability of ultrasonography for the longus colli in asymptomatic subjects. Man Ther 2009;14:421-6.

55 Wallwork TL, Hides JA, Stanton WR. Intrarater and interrater reliability of assessment of lumbar multifidus muscle thickness using rehabilitative ultrasound imaging. J Orthop Sports Phys Ther 2007;37:608-12.

56 Li T, Higgins JPT, Deeks JJ. Chapter 5: Collecting data. In: Higgins JPT, Thomas J, Chandler J, eds. Cochrane Handbook for systematic reviews of interventions. London: Cochrane, 2019.

57 McKenzie JE, Brennan SE, Ryan RE. Chapter 3: Defining the criteria for including studies and how they will be grouped for the synthesis. Draft version (29 January 2019) for inclusion. In: Higgins JPT, Thomas J, Chandler J, eds. Cochrane Handbook for systematic reviews of interventions. London: Cochrane, 2019.

58 Wells GA, Shea B, O'Connell D, et al. The Newcastle-Ottawa scale (NOS) for assessing the quality of nonrandomised studies in meta-analyses. Ottawa Hospital Research Institute. http://www. ohri.ca/programs/clinical_epidemiology/oxford.asp. (cited 2019, 12/04/2019)

59 Seehra J, Pandis N, Koletsi D, et al. Use of quality assessment tools in systematic reviews was varied and inconsistent. J Clin Epidemiol 2016:69:179-84.

60 Quigley JM, Thompson JC, Halfpenny NJ, et al. Critical appraisal of nonrandomized studies-A review of recommended and commonly used tools. J Eval Clin Pract 2019;25:44-52.

61 Luchini C, Stubbs B, Solmi M, et al. Assessing the quality of studies in meta-analyses: advantages and limitations of the Newcastle Ottawa scale. WJMA 2017:5:80-4.

62 Hootman JM, Driban JB, Sitler MR, et al. Reliability and validity of three quality rating instruments for systematic reviews of observational studies. Res Syn Meth 2011;2:110-8.

63 McKenzie JE, Brennan SE, Ryan RE. Chapter 9: Summarizing study characteristics and preparing for synthesis. In: Higgins JPT, Thomas J, Chandler J, eds. Cochrane Handbook for systematic reviews of interventions. London: Cochrane, 2008.

64 López-López JA, Page MJ, Lipsey MW, et al. Dealing with effect size multiplicity in systematic reviews and meta-analyses. Res Syn Meth 2018;9:336-51 https://doi.org/

65 Higgins JPT, Thompson SG, Deeks JJ, et al. Measuring inconsistency in meta-analyses. BMJ 2003;327:557-60.
66 Higgins JPT, Thompson SG. Quantifying heterogeneity in a metaanalysis. Stat Med 2002;21:1539-58.

67 McKenzie JE, Brennan SE. Chapter 12: Synthesizing and presenting findings using other methods. In: Higgins JPT, Thomas J, Chandler J, eds. Cochrane Handbook for systematic reviews of interventions. London: Cochrane, 2008.

68 Deeks JJ, Higgins JPT, Altman DG. Chapter 10: Analysing data and undertaking meta-analyses. In: Higgins JPT, Thomas J, Chandler J, eds. Cochrane Handbook for systematic reviews of interventions. London: Cochrane, 2008.

69 Borenstein M, Higgins JPT. Meta-Analysis and subgroups. Prev Sci 2013;14:134-43.

70 Popay J, Roberts H, Sowden A, et al. Guidance on the conduct of narrative synthesis in systematic reviews: a product from the ESRC methods programme. Lancaster, UK: Lancaster University, 2006.

71 Ryan R. Cochrane Consumers and Communication Review Group. 'Cochrane Consumers and Communication Review Group: data synthesis and analysis, 2013. Available: http://cccrg.cochrane.org [Accessed 30 May 2019].

72 Page MJ, Higgins JPT, Sterne JAC. Chapter 13: Assessing risk of bias due to missing results in a synthesis. In: Higgins JPT, Thomas $\mathrm{J}$, Chandler J, eds. Cochrane Handbook for systematic reviews of interventions. London: Cochrane, 2008.

73 Guyatt G, Oxman AD, AkI EA, et al. Grade guidelines: 1. Introduction-GRADE evidence profiles and summary of findings tables. J Clin Epidemiol 2011;64:383-94.

74 Schünemann H, Brożek J, Guyatt G, eds. Handbook for grading the quality of evidence and the strength of recommendations using the GRADE approach, 2013. https://gdt.gradepro.org/app/handbook/ handbook.html

75 Schünemann AHJ, Higgins JPT, Vist GE. Chapter 14 : Completing 'Summary of findings ' tables and grading the certainty of the evidence. In: Higgins JPT, Thomas J, Chandler J, eds. Cochrane Handbook for systematic reviews of interventions. London: Cochrane, 2008.

76 Balshem H, Helfand M, Schünemann HJ, et al. Grade guidelines: 3. rating the quality of evidence. J Clin Epidemiol 2011;64:401-6.

77 Guyatt GH, Oxman AD, Vist G, et al. Grade guidelines: 4. rating the quality of evidence-study limitations (risk of bias). J Clin Epidemiol 2011;64:407-15. 